\title{
Former very preterm infants show alterations in plasma amino acid profiles at a preschool age
}

\author{
Anna Posod', Susanne Müller', Irena Odri Komazec1,2, Daniel Dejaco³, Ulrike Pupp Peglow', Elke Griesmaier', Sabine \\ Scholl-Bürgi ${ }^{4}$, Daniela Karall ${ }^{4}$ and Ursula Kiechl-Kohlendorfer ${ }^{1}$
}

BACKGROUND: Amino acid analysis is a valuable tool for cardiovascular risk assessment. Preterm infants display plasma amino acid changes in the newborn period. Whether these changes persist is unknown to date. The aim of this study was to assess whether former very preterm infants (VPI) show alterations in amino acid patterns indicative of an unfavorable cardiovascular risk profile at a preschool age.

METHODS: From 5-7 y-old children born at term or $<32$ wk gestation (VPI) were included in the study. Plasma amino acid concentrations were determined after an overnight fast.

RESULTS: 29 former term infants and 79 former VPI were included in the study. Former VPI showed changes in various plasma amino acids including glutamine, arginine, citrulline, tryptophan, glutamate, ornithine, and taurine. Branched-chain amino acids were lower, alanine/lysine ratios significantly higher in the preterm population.

CONCLUSION: Former VPI show altered plasma amino acid profiles indicative of a dualistic cardiovascular risk profile (e.g., potentially beneficial elevations in citrulline, arginine, glutamine, and tryptophan, but also raised alanine/lysine ratios, low ornithine and taurine levels) at a preschool age. Whether this is associated with an adverse cardiovascular outcome has to be addressed by future studies. Long-term cardiometabolic follow-up of VPI might be warranted.

$\mathrm{P}$ reterm birth is a problem of great global relevance. Worldwide, 15 million babies are born preterm every year, accounting for $\sim 11 \%$ of all deliveries (1). Due to advances in perinatal care, survival rates of preterm infants have markedly increased in the last decades. As an increasing proportion of former preterm infants are now reaching adulthood, new health challenges arise (2). A growing body of evidence suggests that prematurity is associated with an unfavorable cardiovascular risk profile in adolescent and adult life (3). Our research group has recently shown that major modifiable markers of an increased cardiovascular risk (elevated blood pressure, raised blood glucose levels and lipid alterations) are present in former very preterm infants (VPI) even at a preschool age and that former VPI display decreased elastic properties of the descending abdominal aorta $(4,5)$. In addition to traditional risk factors, metabolic profiling including plasma amino acid analyses and amino acid-derived scoring systems are now being frequently used as a supplementary investigative tool for cardiovascular risk assessment $(6,7)$. Raised total branched-chain amino acid concentrations (BCAA) and a weighted score based on tyrosine, phenylalanine and isoleucine levels (diabetes-predictive amino acid, DM-AA score) have been shown to be predictive of an adverse cardiovascular outcome in adult low- and high-risk populations (8-12). Data in pediatric cohorts, however, are sparse. Due to immaturity of various enzymatic pathways, plasma amino acid patterns in preterm infants differ from those in term-born controls in the neonatal period (13). If these alterations persist until childhood and contribute to the unfavorable cardiovascular risk profile observed in former preterm infants is unknown to date. The aim of this study was to assess whether very preterm-born children show changes in plasma amino acid profiles at a preschool age. A particular focus was laid on amino acid alterations linked to cardiovascular health and disease.

\section{METHODS}

\section{Study Design and Population}

This study is part of the clinical trial "Preterm infants and early markers for an increased risk of cardiovascular disease", which was carried out at the Department of Pediatrics, Medical University of Innsbruck/Innsbruck University Hospital, Austria, from May 2012 to March 2015. We investigated a group of former VPI born between 1 January 2007 and 31 July 2009 with a gestational age of $<32$ wk, who were invited to a routine preschool visit at our preterm follow-up clinic, and a control group of same-aged children born at term who were recruited through regional kindergartens or while undergoing routine preoperative screening for common surgical procedures at Innsbruck University Hospital. None of the subjects had congenital malformations, chromosomal abnormalities or known inborn errors of metabolism. All children undergoing fasting blood sampling at study visit were assessed for eligibility for plasma amino acid analysis.

\section{Perinatal Characteristics}

Basic perinatal data for each subject were either drawn from the routine VPI follow-up database at our institution or the "Mutter-KindPass" records, the official Austrian pregnancy and early childhood

'Pediatrics II (Neonatology), Department of Pediatrics, Medical University of Innsbruck, Innsbruck, Austria; ${ }^{2}$ Pediatrics III (Pediatric Cardiology), Department of Pediatrics, Medical University of Innsbruck, Innsbruck, Austria; ${ }^{3}$ Department of Otorhinolaryngology, Head and Neck Surgery, Medical University of Innsbruck, Innsbruck, Austria; ${ }^{4}$ Pediatrics I (Inherited Metabolic Disorders), Department of Pediatrics, Medical University of Innsbruck, Innsbruck, Austria. Correspondence: Ursula Kiechl-Kohlendorfer (ursula.kohlendorfer@i-med.ac.at)

Received 3 October 2016; accepted 30 December 2016; advance online publication 22 March 2017. doi:10.1038/pr.2017.24 


\section{Articles Posodetal.}

medical record book. Remaining data were inquired at study visit (see below). Birth weight z-scores were calculated for every subject by means of the Fenton 2013 Growth Calculator for Preterm Infants (available from http://www.peditools.org/fenton2013/). Classification of smoking during pregnancy was based on selfreporting by mothers. Maternal educational status was classified as $<12$ y or $\geq 12 \mathrm{y}$. Infant feeding was categorized as "mainly breastfed", if the content of mothers milk exceeded $50 \%$ in the first month of life. Data on duration of parenteral nutrition and respiratory support in the VPI cohort was extracted from the routine VPI followup database and discharge notes. Prolonged parenteral nutrition was defined as $>10 \mathrm{~d}$. Respiratory support was graded by invasiveness of measure taken; categorization followed the most invasive mode applied in each subject.

\section{Study Visit}

All examinations were performed between $8 \mathrm{AM}$ and $10 \mathrm{AM}$ by specifically trained personnel. Current weight was measured by means of calibrated medical precision scales; current height was determined by a Harpenden stadiometer. BMI was calculated as weight in kilograms divided by height in meters squared. BMI z-scores were calculated for each study participant by means of a reference data set (14).

Childhood nutrition was categorized as unfavorable, neutral or favorable based on a standardized food frequency questionnaire ("What do you eat?" kindly provided by the Robert Koch Institute, Berlin, Germany) (15).

Lithium heparin samples were collected after a minimum overnight fasting period of $8 \mathrm{~h}$. Blood glucose and insulin levels were determined at the Central Institute for Medical and Chemical Laboratory Diagnosis (Innsbruck University Hospital). Homeostasis model assessment index (HOMA-IR) as a surrogate marker of insulin resistance was calculated for each subject as fasting insulin $(\mathrm{mU} / \mathrm{l})$ ) $\times$ fasting glucose $(\mathrm{mg} / \mathrm{dl}))$, / 405. HOMA-IR percentiles were calculated by means of an age- and sex-specific European reference data set (16). Plasma amino acid levels were determined at the Metabolic Laboratory (Pediatrics I, Medical University of Innsbruck, Innsbruck, Austria) by means of a Biochrom 30+ Amino Acid Analyzer Physiological System based on ion exchange chromatography with postcolumn derivatization with ninhydrin (Laborservice Onken, Gründau, Germany) according to the manufacturer's instructions (protocols can be provided immediately upon request). BCAA were calculated as the sum of valine, leucine, and isoleucine concentrations. Fischer's ratio was calculated as the molar ratio of BCAA to aromatic amino acids (phenylalanine, tyrosine). DM-AA scores were calculated as follows: $\mathrm{z}$-score of $\log$ (tyrosine) $+\mathrm{z}$-score of $\log$ (phenylalanine) $+\mathrm{z}$-score of $\log$ (isoleucine) $(10,11)$.

\section{Statistical Analysis}

All statistical analyses were carried out with SPSS for Windows, version 22 (SPSS, Armonk, NY). To assess representativeness of samples, both study cohorts were compared with reference populations (term: SIDS database Tyrol, birth years 2007-2009; preterm: Innsbruck routine VPI follow-up database, birth years 2007-2009) in regard to gender distribution, gestational age, birth weight, and maternal educational status by Pearson's $\chi^{2}$ or Mann-Whitney $U$-test.

Differences in perinatal characteristics and characteristics at study visit between study groups were determined by Pearson's $\chi^{2}$, MannWhitney $U$ - or Student's $t$-test, depending on type and distribution of the variable analyzed.

To assess differences in plasma amino acid concentrations between very preterm and term subjects, logistic regression analysis was used. Input data were logarithmically transformed prior to analyses to account for skewed distributions or heteroscedasticity of the dependent variable. Adjustments for potential confounders were made step-wise: Model A was adjusted for age at examination and sex, model B for all parameters of model A plus birth weight $\mathrm{z}$-score, smoking during pregnancy, maternal educational status, current BMI z-scores, breastfeeding practices, and childhood nutrition profiles. Odds ratios (OR) and respective confidence intervals $(\mathrm{CI})$ were calculated as OR per SD increase.

In order to account for pre-existing alterations in glucose metabolism and their potential influence on DM-AA scores, a subgroup analysis was performed for subjects with HOMA-IR indices above the $75^{\text {th }}$ percentile in both term and very preterm study groups by means of Mann-Whitney $U$-test with Bonferroni correction for multiple comparisons.

Subgroup analyses for prolonged parenteral nutrition and infant feeding in the VPI cohort were conducted with Mann-Whitney $U$-test and Bonferroni correction for multiple comparisons. Subgroup analyses for respiratory support modes in the neonatal period were carried out by means of Kruskal-Wallis test with post hoc MannWhitney $U$-test and Bonferroni correction for multiple comparisons.

\section{Study Approval}

Ethical approval was obtained from the institutional review board of the Medical University of Innsbruck (approval no. AN 4491 305/4.7). Written informed consent was obtained from all legal guardians and verbal consent from all study participants prior to inclusion in the study.

\section{RESULTS \\ Study Population}

About 84 children born very preterm and 34 children born at term were assessed for eligibility. Plasma amino acid profiles were available in 79 former VPI and 30 former term infants. One child born at term had to be excluded from the study due to a previously unknown diagnosis of hyperprolinemia.

In former term infants, sex distribution, birth weight, and maternal educational status were similar to the general Tyrolean birth cohort. Former VPI did not significantly differ from all VPI in the survey area in gestational age, birthweight, sex distribution or maternal educational status.

\section{Population Characteristics}

In children enrolled in the study, sex distribution was similar in both study groups. In former VPI, gestational age and birthweight inherently were significantly lower in comparison to children born at term. Birthweight z-scores did not differ between groups. Smoking and breastfeeding habits as well as maternal educational status were similar in both study groups. In the very preterm study group, BMI and BMI z-scores were significantly lower at study visit and favorable childhood nutrition profiles were less frequently reported in food propensity questionnaires. Unfavorable eating habits were not reported in either study group. HOMA-IR indices were significantly higher in the very preterm population. None of the children were taking medication with a possible impact on amino acid or glucose metabolism. In the VPI cohort, median duration of parenteral nutrition in the neonatal period was $11 \mathrm{~d}$ (minimum $3 \mathrm{~d}$ and maximum $100 \mathrm{~d}$ ). With regard to respiratory support modes applied, $2.6 \%$ of VPI received none, $46.8 \%$ received continuous positive airway pressure by a nasal device, $36.4 \%$ were conventionally ventilated, and $14.2 \%$ required high frequency oscillation ventilation. Details of population characteristics can be found in Table 1 .

\section{Plasma Amino Acid Patterns}

All values measured in both study groups were considered nonpathological for the age group (17). In comparison to children born at term, former VPI had significantly higher glutamine, tryptophan, arginine, citrulline, hydroxproline, cystine, 3 -methylhistidine, and $\beta$-aminoisobutyric acid concentrations 
Table 1. Perinatal characteristics and characteristics at study visit in former term and preterm infants

\begin{tabular}{|c|c|c|}
\hline Characteristic & Term group $(n=29)$ & Preterm group $(n=79)$ \\
\hline Sex, male/female $(n)(\%)$ & $14(48.3) / 15(51.7)$ & $40(50.6) / 39(49.4)$ \\
\hline \multicolumn{3}{|l|}{ Perinatal characteristics } \\
\hline Birth weight, mean (SD) (grams) & $3295(481)$ & $1218(405)^{* * *}$ \\
\hline Birth weight z-score, mean (SD) & $-0.332(0.941)$ & $-0.088(0.899)$ \\
\hline Mainly (>50\%) breastfed, unknown/yes/no (\%) & $6.9 / 72.4 / 20.7$ & $0.0 / 78.5 / 21.5$ \\
\hline \multicolumn{3}{|l|}{ Characteristics at study visit } \\
\hline Age at examination, mean (SD) (years) & $5.6(0.7)$ & $5.4(0.3)$ \\
\hline Current BMI, median (IQR) (kg/m²) & $14.6(1.5)$ & $14.1(1.3)^{*}$ \\
\hline
\end{tabular}

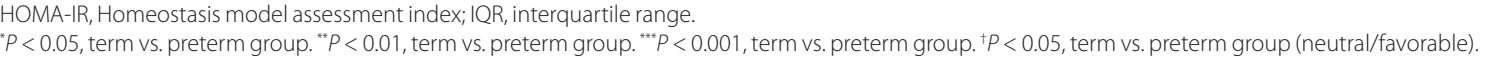

(Table 2). Serine, leucine, glutamate, taurine, ornithine, histidine, asparagine, and aspartate concentrations were significantly lower in the very preterm study group (Table 2). After adjustment for age at examination and sex, statistically significant differences in glutamine concentrations could not be observed any more. Remaining results were similar after adjustment for covariates (see Supplementary Tables S1-S3 online). Representative graphs of plasma amino acid concentrations in term- and very preterm-born study populations are presented in Figure 1.

BCAA concentrations, phenylalanine/tyrosine ratios and Fischer's quotient were lower in the very preterm study group; however these findings did not reach statistical significance after adjustment for covariates (Table 2 and Supplementary Tables S1-S3 online).

Alanine/lysine ratios were significantly higher in former VPI. DM-AA scores were similar in both study groups (Table 2 and Supplementary Table S1 online). HOMA-IR indices above the $75^{\text {th }}$ percentile were observed in 7 term- and in 38 very preterm-born children. A subgroup analysis of DM-AA scores between former VPI with HOMA-IR indices above vs. HOMA-IR indices below the $75^{\text {th }}$ percentile showed no statistically significant differences between groups $(P=0.633$ with adjusted significance level $\alpha=0.017$ ). In addition, no significant differences were observed in DM-AA scores in children with HOMA-IR indices above the $75^{\text {th }}$ percentile between term and very preterm study groups $(P=0.866$ with adjusted significance level $\alpha=0.017$ ).

In the VPI cohort, measured plasma amino acid concentrations and derived indices did not differ between subjects who did or did not receive prolonged parenteral nutrition in the newborn period. Also, no differences were detected between mainly formula- and mainly human milk-fed VPI (see Supplementary Table S2 online).
With regard to respiratory support modes in the neonatal period, no significant differences in plasma amino acid concentrations and derived indices were detected between subjects who received no support, nasal continuous positive airway pressure, conventional ventilation or high frequency oscillation ventilation (see Supplementary Table S3 online).

\section{DISCUSSION}

Amino acids play a crucial role in fetal and neonatal growth and development (18). Due to delays in postnatal feeding and a lack of energy stores developed in the last trimester of pregnancy, preterm infants often suffer from early postnatal catabolism until feeding is established (19). Subsequent suboptimal nutritional supply might have long-lasting effects on metabolism (20). In our study, all amino acid concentrations determined in term- and very preterm-born subjects were within age-appropriate reference ranges, thus not indicating any inborn errors of metabolism (17). However, also high- or low-normal values might be of relevance, as changes in amino acid metabolism in early disease phases are often subtle (17).

The observed alterations in amino acid concentrations in our study affect various metabolic pathways. Overall changes are reconcilable with an underlying accelerated metabolism, which may be attributed to high amino acid usage and/or turnover in the preterm population, potentially resulting from early metabolic imprinting $(21,22)$. In order to corroborate this hypothesis, however, longitudinal measurements and determination of amino acid dynamics are required.

With regard to cardiovascular risk assessment, we observed dualistic alterations in amino acid profiles. In comparison to term-born controls, very preterm subjects had significantly higher plasma concentrations of arginine, which inter alia acts as a nitric oxide donor, and its precursor citrulline (23). Nitric oxide is a key regulator of cardiovascular homeostasis and an 


\section{Articles $\mid$ Posodetal.}

Table 2. Plasma amino acid profiles in children born preterm in comparison to same-aged controls born at term

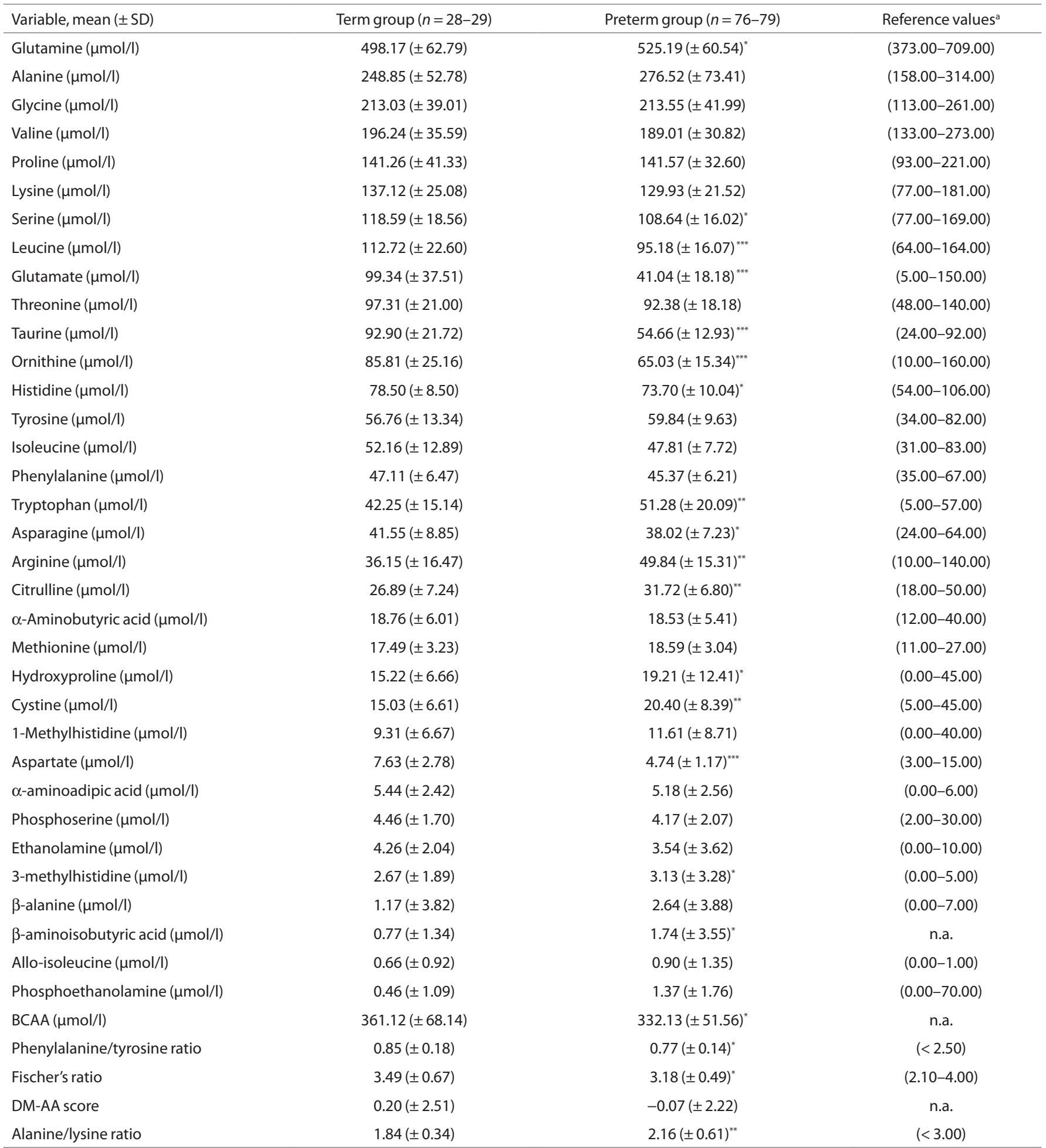

BCAA, branched-chain amino acid concentrations; DM-AA, diabetes-predictive amino acid; n.a., not available/applicable.

aBased on ref. (17). ${ }^{*} P<0.05$ term vs. preterm group. ${ }^{* *} P<0.01$ term vs. preterm group. ${ }^{* * *} P<0.001$ term vs. preterm group.

important protective molecule in the vasculature $(24,25)$. In Zucker diabetic fatty rats, a citrulline-rich diet and subsequent increased arginine availability ameliorated the phenotype of metabolic syndrome, thus suggesting a beneficial effect of relative citrulline- and argininemia (26). One of the quantitatively most relevant catabolic pathways of arginine is its metabolism to ornithine and urea via arginase (27). Interestingly, ornithine concentrations were significantly lower in VPI whereas urea levels did not significantly differ between groups (mean $\pm \mathrm{SD}$, term $23.5 \pm 5.8 \mathrm{mg} / \mathrm{dl}$ vs. preterm $26.0 \pm 6 \mathrm{mg} / \mathrm{dl} ; P=$ 
a

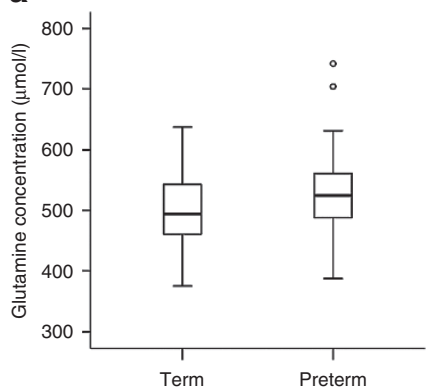

e

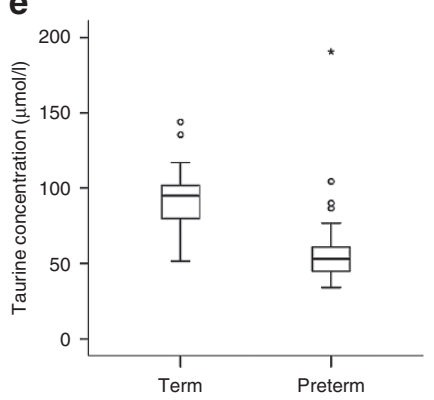

i

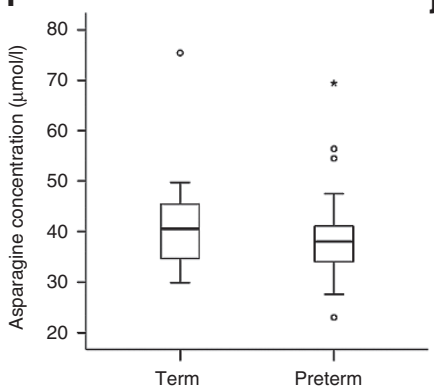

m

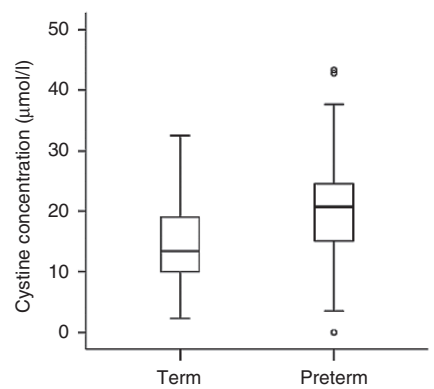

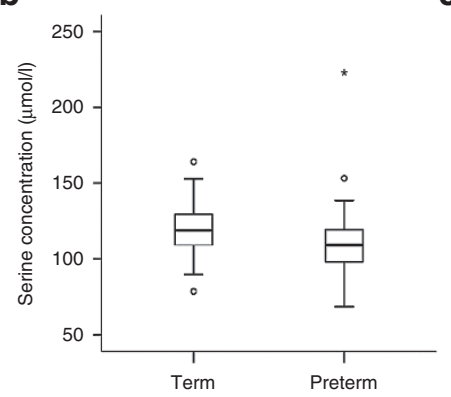

f

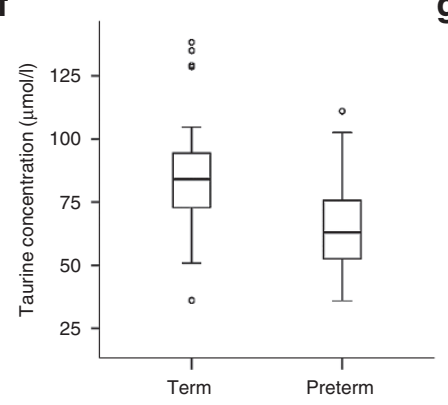

j

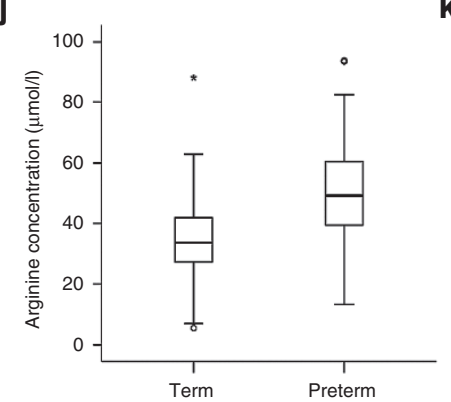

n

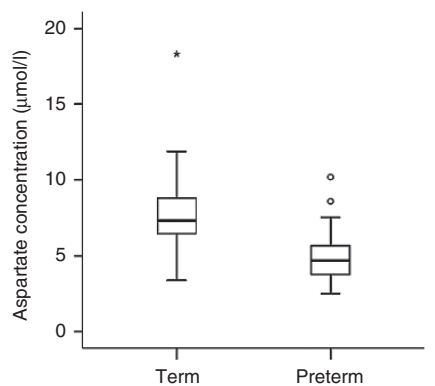

C

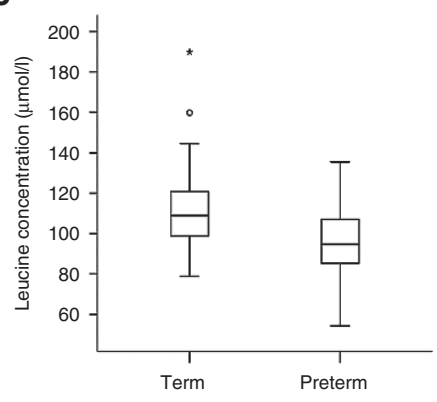

g

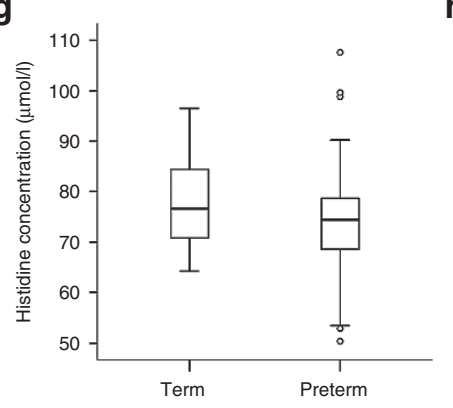

k

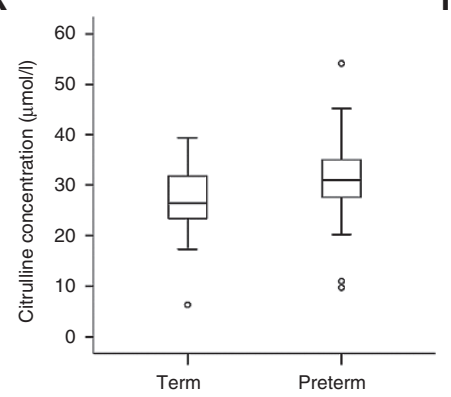

0

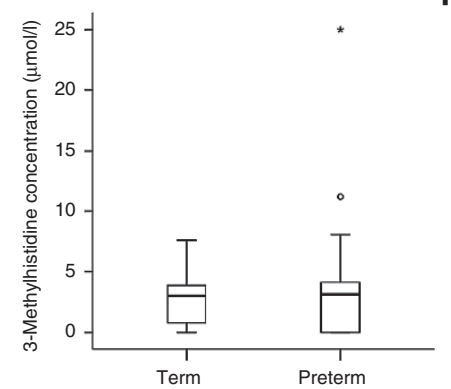

d

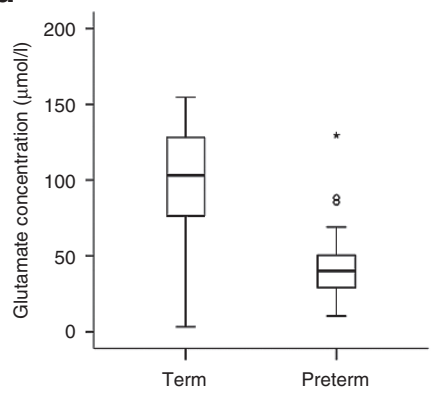

h

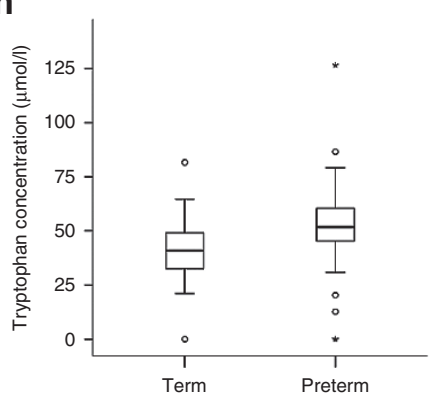

I

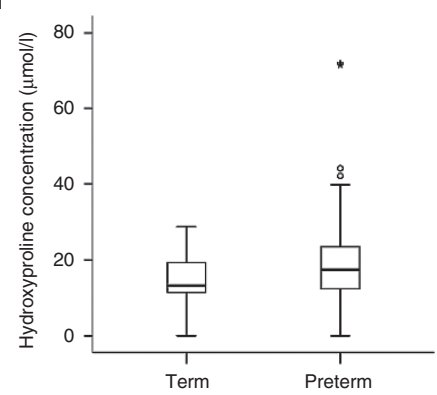

p

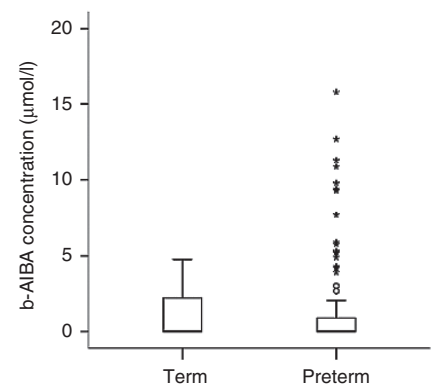

Figure 1. Plasma amino acid concentrations. Changes in glutamine (a), serine (b), leucine (c), glutamate (d), taurine (e), ornithine (f), histidine (g),

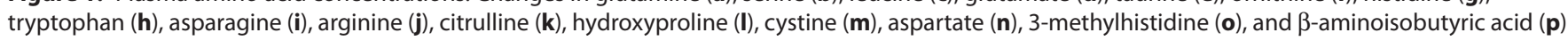
concentrations in former very preterm infants in comparison to term-born controls. Plasma amino acid concentrations are presented in $\mu$ mol/l; whiskers represent 1.5-fold interquartile ranges; circles represent outliers; and asterisks extreme outliers. Number of subjects per group: term $n=29$, preterm $n=$ 79. b-AIBA, $\beta$-aminoisobutyric acid.

0.124, Mann-Whitney $U$-test, not shown). Ornithine can be further metabolized to polyamines (putrescine, spermidine, and spermine), which are involved in various cellular functions including cell proliferation and differentiation (27). Even though the catalyzing enzyme, ornithine decarboxylase, is rate-controlling for this step, also ornithine availability is considered a limiting factor (28). As deregulation of polyamine pathways has been implicated in various pathological conditions such as stroke, and recent data suggest cardioprotection by spermidine supplementation $(27,29)$, longitudinal assessment of the relevance of this finding in regard to future cardiovascular health of VPI seems advisable.

In addition, former VPI displayed elevated glutamine and diminished glutamate plasma concentrations. Cheng et al. have recently demonstrated an inverse correlation of glutamine and a direct correlation of glutamate levels with adverse metabolic 


\section{Articles | Posodetal.}

traits (6). In a multicenter randomized trial of dietary interventions, baseline glutamate was associated with an increased and glutamine-to-glutamate ratio with a decreased risk of composite cardiovascular disease, consisting of nonfatal stroke, nonfatal myocardial infarction, or cardiovascular death (30). Also, higher tryptophan plasma concentrations were measured in former VPI. A large Norwegian survey has recently reported an inverse correlation of tryptophan levels with allcause and cardiovascular mortality (31). The observed changes in VPI in our study might thus be associated with a favorable cardiovascular outcome. Especially with regard to tryptophan, however, additional studies are direly needed in order to determine whether the observed elevation in tryptophan is due to (cardiovascularly beneficial) decreased tryptophan degradation (32). Moreover, phenylalanine/tyrosine ratios were lower in former VPI, suggesting a lower level of systemic inflammation potentially associated with cardiovascular disease $(33,34)$. Significance, however, was lost after covariate adjustment.

Taurine plasma concentrations were significantly lower in VPI in all statistical models applied. This is of interest, as taurine supplementation has been reported to reduce hypertension and stroke in experimental animal models as well as exert antioxidative effects in healthy male adults $(35,36)$. In addition, urinary taurine excretion was shown to be inversely correlated with age-adjusted mortality rates of coronary heart disease (36). Whether former VPI might benefit from taurine supplementation remain to be investigated.

Fischer's ratio was lower in very preterm subjects, indicating a relative elevation of the aromatic amino acids phenylalanine and tyrosine. The relevance of this finding with regard to cardiovascular disease is ambiguous. In an experimental model of voluntary long-term exercise, Fischer's ratio was significantly lower in physically active rodents, which also had a higher skeletal muscle weight and a lower white adipose tissue and liver weight (37). In opposition, Hakuno et al. have previously reported a correlation of Fischer's ratio with cardiac function and prognostic markers in patients with systolic heart failure (38). In patients with pulmonary hypertension, Fischer's ratio has also been shown to decrease in proportion with clinical disease severity (39). The lower Fischer's ratio observed in former VPI in our study might thus be either a favorable or an adverse trait, necessitating follow-up assessment. Statistical significance, however, was not attained after adjustment for covariates.

A growing body of evidence suggests an association of BCAA concentrations with unfavorable cardiometabolic phenotypes in both adult and pediatric cohorts $(7,12,40-42)$. Interestingly, even though we have recently shown that former VPI have an adverse classical and echocardiographic cardiovascular risk profile $(4,5)$, BCAA concentrations were lower in former VPI than in term-born controls in this study. However, BCAA plasma concentrations are known to be closely related to visceral fat accumulation $(8,9)$, which seems to be of minor relevance in our study population, as former VPI had significantly lower BMI and BMI z-scores at study visit than term-born controls. After adjustment for covariates, significance was not attained.

The DM-AA score was chosen as a cardiovascular risk indicator, as it has previously been shown to predict diabetes onset as well as cardiovascular events even in an asymptomatic population $(10,11)$. In our study, DM-AA scores did not significantly differ between groups. However, this score is based on fasting plasma levels of the BCAA isoleucine as well as the aromatic amino acids tyrosine and phenylalanine, all of which have been shown to be raised in obese subjects contrasting our lean very preterm cohort (43). As branched chain and aromatic amino acids have been linked to insulin ineffectiveness (43), a subgroup analysis of subjects with increased vs. normal HOMA-IR indices was also carried out in order to rule out a potential influence of pre-existing alterations in glucose metabolism. This analysis, however, yielded similar results.

Of interest, even though within a normal range $(<3: 1)$, alanine/lysine ratios as a marker of intra/extramitochondrial metabolism were significantly higher in the very preterm study cohort, thus potentially indicating incipient mitochondrial dysfunction. As mitochondrial disease is commonly associated with cardiomyopathy or diabetes mellitus, and dysfunctional mitochondria are known to contribute to cardiovascular and metabolic disease, this finding might be linked to an adverse cardiovascular outcome (44-46).

A main limitation of our study is that amino acid concentrations were only determined at a single time point in early childhood. Whether alterations pre-existed in the study population and/or persist later in life is unknown to date. It is also possible that the metabolic changes observed and preterm birth as such can be ascribed to a currently unknown-common denominator. Furthermore, it remains to be elucidated whether changes result from true epigenetic modifications or merely represent altered body composition in the VPI cohort. On top, it is open to question whether the changes noted are associated with adverse health outcomes, and whether alterations might be preventable by specific health interventions or adaptations in perinatal care policies. In order to unravel underlying etiopathogenetic mechanisms, assess amino acid dynamics and shed light on potential consequences, both prospective trials starting in the neonatal period and extensive long-term studies are required. Regardless, reconceptualization of preterm follow-up programs with particular focus on metabolic traits might be advisable.

To summarize, in comparison to children born at term, former VPI display altered plasma amino acid profiles at a preschool age. In contrast to our previous findings, indicating an unfavorable classical cardiovascular risk profile, amino acid changes are ambiguous. Alterations known to be associated with both beneficial, but also unfavorable cardio-metabolic traits were observed in former VPI. Prospective studies with larger cohorts are direly needed to scrutinize amino acid dynamics and to clarify whether changes are causally linked to cardiovascular health and disease or whether they represent mere compensatory mechanisms. Longer-term routine 
follow-up of former VPI in regard to cardiometabolic risk factors might be warranted.

\section{SUPPLEMENTARY MATERIAL}

Supplementary material is linked to the online version of the paper at http://www.nature.com/pr

\section{ACKNOWLEDGMENTS}

The authors thank Katrin Kager, for her logistic and motivational support throughout the trial. The authors also thank Ulrike Eichinger-Öttl and Claudia Ertl, for performing all chromatographic analyses and kindly sharing their methodological expertise. Finally, the authors thank all children and their families for participating in and committing to our study.

\section{STATEMENT OF FINANCIAL SUPPORT}

This work was supported by funds of the Oesterreichische Nationalbank (Oesterreichische Nationalbank, Anniversary Fund, project no. 14570) and Oesterreichischer Herzfonds. Ursula Kiechl-Kohlendorfer was also supported by the excellence initiative (Competence Centers for Excellent TechnologiesCOMET) of the Austrian Research Promotion Agency FFG:"Research Center of Excellence in Vascular Ageing-Tyrol, VASCage" (K-Project no. 843536) funded by BMVIT, BMWFW, Wirtschaftsagentur Wien, and Standortagentur Tirol.

Disclosure: The authors declare that they have no conflicts of interest.

\section{REFERENCES}

1. Harrison MS, Goldenberg RL. Global burden of prematurity. Semin Fetal Neonatal Med 2016;21:74-9.

2. Bayman E, Drake AJ, Piyasena C. Prematurity and programming of cardiovascular disease risk: a future challenge for public health? Archives of Disease in Childhood Fetal and Neonatal Edition 2014;99:F510-4.

3. Sipola-Leppänen M, Kajantie E. Should we assess cardiovascular risk in young adults born preterm? Curr Opin Lipidol 2015;26:282-7.

4. Posod A, Odri Komazec I, Kager K, Pupp Peglow U, Baumgartner D, Kiechl-Kohlendorfer U. PS-104 early markers of an increased cardiovascular risk in former preterm tyrolean preschoolers-preliminary data. Archives of disease in childhood 2014;99:A148.

5. Odri Komazec I, Posod A, Schwienbacher M, et al. Aortic elastic properties in preschool children born preterm. Arterioscler Thromb Vasc Biol 2016;36:2268-74.

6. Cheng S, Rhee EP, Larson MG, et al. Metabolite profiling identifies pathways associated with metabolic risk in humans. Circulation 2012;125:2222-31.

7. Rhee EP, Gerszten RE. Metabolomics and cardiovascular biomarker discovery. Clin Chem 2012;58:139-47.

8. Shah SH, Crosslin DR, Haynes CS, et al. Branched-chain amino acid levels are associated with improvement in insulin resistance with weight loss. Diabetologia 2012;55:321-30.

9. Newgard CB, An J, Bain JR, et al. A branched-chain amino acid-related metabolic signature that differentiates obese and lean humans and contributes to insulin resistance. Cell Metab 2009;9:311-26.

10. Wang TJ, Larson MG, Vasan RS, et al. Metabolite profiles and the risk of developing diabetes. Nat Med 2011;17:448-53.

11. Magnusson M, Lewis GD, Ericson U, et al. A diabetes-predictive amino acid score and future cardiovascular disease. Eur Heart J 2013;34:1982-9.

12. Mangge H, Zelzer S, Prüller F, et al. Branched-chain amino acids are associated with cardiometabolic risk profiles found already in lean, overweight, and obese young. J Nutr Biochem 2016;32:123-7.

13. Rigo J, Senterre J. Significance of plasma amino acid pattern in preterm infants. Biol Neonate 1987;52 Suppl 1:41-9.

14. Kromeyer-Hauschild K, Wabitsch M, Kunze D, Gellert F, Geiß HC, Hesse V. Percentiles of body mass index in children and adolescents evaluated from different regional German cohorts. Monatsschrift für Kinderheilkunde 2001:807-18.

15. Truthmann J, Mensink GB, Richter A. Relative validation of the KiGGS food frequency questionnaire among adolescents in Germany. Nutr J 2011;10:133.

16. Peplies J, Jiménez-Pavón D, Savva SC, et al.; IDEFICS consortium. Percentiles of fasting serum insulin, glucose, HbAlc and HOMA-IR in prepuber- tal normal weight European children from the IDEFICS cohort. Int J Obes (Lond) 2014;38 Suppl 2:S39-47.

17. Duran M. Amino acids. In: Blau N, Duran M, Gibson KM, eds. Laboratory Guide to the Methods in Biochemical Genetics. Berlin Heidelberg: Springer Verlag, 2008:53-89.

18. Corpeleijn WE, van den Akker CH, Roelants JA, van Goudoever JB. How proteins improve the development of preterm infants. Nestle Nutr Workshop Ser Pediatr Program 2011;68:33-45; discussion 45-8.

19. de Boo HA, Harding JE. Protein metabolism in preterm infants with particular reference to intrauterine growth restriction. Archives of Disease in Childhood Fetal and Neonatal Edition 2007;92:F315-9.

20. Barker DJ. Fetal and infant origins of adult disease. Monatsschrift Kinderheilkunde: Organ der Deutschen Gesellschaft fur Kinderheilkunde 2001;149:5.

21. Pencharz PB, Masson M, Desgranges F, Papageorgiou A. Total-body protein turnover in human premature neonates: effects of birth weight, intrauterine nutritional status and diet. Clin Sci (Lond) 1981;61:207-15.

22. Piyasena C, Cartier J, Khulan B, et al. Dynamics of DNA methylation at IGF2 in preterm and term infants during the first year of life: an observational study. Lancet 2015;385 Suppl 1:S81.

23. Wu G, Morris SM Jr. Arginine metabolism: nitric oxide and beyond. Biochem J 1998;336 (Pt 1):1-17.

24. Oliveira-Paula GH, Lacchini R, Tanus-Santos JE. Clinical and pharmacogenetic impact of endothelial nitric oxide synthase polymorphisms on cardiovascular diseases. Nitric Oxide 2017;63:39-51.

25. Förstermann U, Münzel T. Endothelial nitric oxide synthase in vascular disease: from marvel to menace. Circulation 2006;113:1708-14.

26. Wu G, Collins JK, Perkins-Veazie P, et al. Dietary supplementation with watermelon pomace juice enhances arginine availability and ameliorates the metabolic syndrome in Zucker diabetic fatty rats. J Nutr 2007;137: 2680-5.

27. Popolo A, Adesso S, Pinto A, Autore G, Marzocco S. L-Arginine and its metabolites in kidney and cardiovascular disease. Amino Acids 2014;46:2271-86.

28. Li H, Meininger CJ, Bazer FW, Wu G. Intracellular sources of ornithine for polyamine synthesis in endothelial cells. Amino Acids 2016;48:2401-10.

29. Eisenberg T, Abdellatif M, Schroeder S, et al. Cardioprotection and lifespan extension by the natural polyamine spermidine. Nat Med 2016;22:142838.

30. Zheng Y, Hu FB, Ruiz-Canela M, et al. Metabolites of glutamate metabolism are associated with incident cardiovascular events in the PREDIMED PREvencion con DIeta MEDiterranea (PREDIMED) Trial. Journal of the American Heart Association 2016;5:e003755.

31. Zuo H, Ueland PM, Ulvik A, et al. Plasma biomarkers of inflammation, the Kynurenine pathway, and risks of all-cause, cancer, and cardiovascular disease mortality: the Hordaland health study. Am J Epidemiol 2016;183:24958.

32. Polyzos KA, Ketelhuth DF. The role of the Kynurenine pathway of tryptophan metabolism in cardiovascular disease. An emerging field. Hamostaseologie 2015;35:128-36.

33. Wannemacher RW Jr, Klainer AS, Dinterman RE, Beisel WR. The significance and mechanism of an increased serum phenylalanine-tyrosine ratio during infection. Am J Clin Nutr 1976;29:997-1006.

34. Murr C, Grammer TB, Meinitzer A, Kleber ME, März W, Fuchs D. Immune activation and inflammation in patients with cardiovascular disease are associated with higher phenylalanine to tyrosine ratios: the Ludwigshafen risk and cardiovascular health study. J Amino Acids 2014;2014:783730.

35. Katakawa M, Fukuda N, Tsunemi A, et al. Taurine and magnesium supplementation enhances the function of endothelial progenitor cells through antioxidation in healthy men and spontaneously hypertensive rats. Hypertens Res 2016;39:848-56.

36. Yamori Y, Taguchi T, Hamada A, Kunimasa K, Mori H, Mori M. Taurine in health and diseases: consistent evidence from experimental and epidemiological studies. J Biomed Sci 2010;17 Suppl 1:S6.

37. Takeshita $\mathrm{H}$, Horiuchi M, Izumo K, et al. Long-term voluntary exercise, representing habitual exercise, lowers visceral fat and alters plasma amino acid levels in mice. Environ Health Prev Med 2012;17:275-84. 


\section{Articles Posodetal.}

38. Hakuno D, Hamba Y, Toya T, Adachi T. Plasma amino acid profiling identifies specific amino acid associations with cardiovascular function in patients with systolic heart failure. PLoS One 2015;10: e0117325.

39. Yanagisawa R, Kataoka M, Inami T, et al. Usefulness of circulating amino acid profile and Fischer ratio to predict severity of pulmonary hypertension. Am J Cardiol 2015;115:831-6.

40. McCormack SE, Shaham O, McCarthy MA, et al. Circulating branchedchain amino acid concentrations are associated with obesity and future insulin resistance in children and adolescents. Pediatr Obes 2013;8: $52-61$.

41. Würtz P, Havulinna AS, Soininen $P$, et al. Metabolite profiling and cardiovascular event risk: a prospective study of 3 population-based cohorts. Circulation 2015;131:774-85.
42. Ho JE, Larson MG, Ghorbani A, et al. Metabolomic profiles of body mass index in the Framingham heart study reveal distinct cardiometabolic phenotypes. PLoS One 2016;11:e0148361.

43. Felig P, Marliss E, Cahill GF Jr. Plasma amino acid levels and insulin secretion in obesity. N Engl J Med 1969;281:811-6.

44. Gamboa JL, Billings FTt, Bojanowski MT, et al. Mitochondrial dysfunction and oxidative stress in patients with chronic kidney disease. Physiological Reports 2016;4:e12780.

45. Duicu OM, Lighezan R, Sturza A, et al. Assessment of mitochondrial dysfunction and monoamine oxidase contribution to oxidative stress in human diabetic hearts. Oxid Med Cell Longev 2016;2016:8470394.

46. Chinnery PF. Mitochondrial disorders overview. In: Pagon RA, Adam MP, Ardinger $\mathrm{HH}$, et al., eds. GeneReviews(R). Seattle, WA: University of Washington, Seattle; 1993-2017. 